\title{
Studies of low-energy $\mathrm{K}^{-}$nuclear interactions by AMADEUS
}

R. Del Grande ${ }^{1,2, a}$, M. Bazzi ${ }^{1}$, G. Belloti ${ }^{3}$, A. M. Bragadireanu ${ }^{4}$, D. Bosnar ${ }^{5}$, A. D. Butt ${ }^{3,6}$, M. Cargnelli ${ }^{7}$, C. Curceanu ${ }^{1}$, L. De Paolis ${ }^{1,2}$, L. Fabbietti ${ }^{8,9}$, C. Fiorini ${ }^{3,6}$, F. Ghio ${ }^{10,11}$, C. Guaraldo ${ }^{1}$, R. S Hayano ${ }^{12}$, M. Iliescu ${ }^{1}$, M. Imasaki ${ }^{13}$, P. Levi Sandri ${ }^{1}$, J. Marton ${ }^{7}$, M. Miliucci ${ }^{1,2}$, P. Moskal ${ }^{14}$, S. Okada ${ }^{13}$, D. Pietreanu ${ }^{4}$, K. Piscicchia ${ }^{1}, 15$, A. Scordo ${ }^{1}$, H. Shi ${ }^{1}$, M. Silarski ${ }^{14}$, D. L. Sirghi ${ }^{1,4}$, F. Sirghi ${ }^{1,4}$, M. Skurzok ${ }^{14}$, A. Spallone ${ }^{1}$, O. Vazquez Doce ${ }^{8,9}$, E. Widmann ${ }^{7}$, S. Wycech ${ }^{16}$, and J. Zmeskal ${ }^{7}$

${ }^{1}$ INFN Laboratori Nazionali di Frascati, Frascati, Rome, Italy

${ }^{2}$ Università degli Studi di Roma Tor Vergata, Rome, Italy

${ }^{3}$ Politecnico di Milano, Dip. di Elettronica, Informazione e Bioingegneria, Milano, Italy

${ }^{4}$ Horia Hulubei National Institute of Physics and Nuclear Engineering, Magurele, Romania

${ }^{5}$ University of Zagreb, Zagreb, Croatia

${ }^{6}$ INFN Sezione di Milano, Milano, Italy

${ }^{7}$ Stefan-Meyer-Institut für Subatomare Physik, Wien, Austria

${ }^{8}$ Excellence Cluster 'Origin and Structure of the Universe', Garching, Germany

${ }^{9}$ Physik Department E12, Technische Universität München, Garching, Germany

${ }^{10}$ INFN Sezione di Roma I, Rome, Italy

${ }^{11}$ Istituto Superiore di Sanità, Rome, Italy

${ }^{12}$ The University of Tokyo, Tokyo, Japan

${ }^{13}$ RIKEN, The Institute of Physics and Chemical Research, Saitama, Japan

${ }^{14}$ Institute of Physics, Jagiellonian University, Kracow, Poland

${ }^{15}$ Museo Storico della Fisica e Centro Studi e Ricerche Enrico Fermi, Rome, Italy

${ }^{16}$ National Centre for Nuclear Research, Warsaw, Poland

\begin{abstract}
The goal of the AMADEUS experiment is to shed light on unsolved fundamental issues in the non-perturbative strangeness QCD sector through the study of lowenergy $\mathrm{K}^{-}$hadronic interactions with light nuclear targets. The main open questions are the controversial nature of the $\Lambda(1405)$ state, which is investigated in hyperon-pion correlation studies, and the possible existence of exotic antikaon multi-nucleon clusters, whose search in $\mathrm{K}^{-}$induced reactions is intimately related to the studies of the $\mathrm{K}^{-}$multi-nucleon absorption processes in hyperon-nucleon/nucleus channels. The DAФNE collider at the INFN-LNF provides unique monochromatic low-momentum kaons from the $\phi$ meson decay almost at-rest, suitable for the AMADEUS studies. The KLOE detector is exploited as an active target, in order to obtain excellent acceptance and resolution data for $\mathrm{K}^{-}$ nuclear capture on $\mathrm{H},{ }^{4} \mathrm{He},{ }^{9} \mathrm{Be}$ and ${ }^{12} \mathrm{C}$, both at-rest and in-flight.
\end{abstract}

a e-mail: raffaele.delgrande@lnf.infn.it 


\section{Introduction}

The AMADEUS (Anti-kaonic Matter At DAФNE: An Experiment with Unraveling Spectroscopy) [1] collaboration aims to provide unique quality data of low-energy $\mathrm{K}^{-}$hadronic interactions in light nuclei (e.g. $\mathrm{H},{ }^{4} \mathrm{He},{ }^{9} \mathrm{Be}$ and ${ }^{12} \mathrm{C}$ ) in order to afford experimental constraints on the non-perturbative QCD in the strangeness sector. AMADEUS takes advantage of the low momentum (about 127 $\mathrm{MeV} / \mathrm{c}$ ), almost monochromatic, charged kaons provided by the decay of $\phi$ mesons at-rest at the DAФNE factory [2]. The analyses presented here refers to the data acquired by the KLOE [3] collaboration during the 2004-2005 data taking campaign.

Chiral perturbation theory is not applicable in the $u d s \mathrm{SU}(3)$ flavour sector due to the existence of broad hyperon resonances below the $\overline{\mathrm{K}} \mathrm{N}$ threshold, rendering the perturbative approach inappropriate. The non-perturbative chiral SU(3) dynamics [4-7] and the phenomenological potential approach [8,9] are the main theoretical tools to overcome the difficulty. In both cases the $\overline{\mathrm{K}}$ dynamics above threshold is well described because the two models are constrained to the scattering data but the sub-threshold extrapolations are in strong disagreement $[10,11]$. Chiral models predict significantly weaker $\bar{K} N$ interaction with respect to phenomenological potentials approach. The position of the $\Lambda(1405)(\mathrm{I}=0)$ state, which is experimentally observed through the decay into $(\Sigma \pi)^{0}$ channels, seems to depend on both the observed decay channel and on the production mechanism. Chiral unitary models predict the emerging of two poles contributing to the $\Lambda(1405)$ line-shape, with a high mass pole (located at about $1420 \mathrm{MeV}$ ) strongly coupled to the $\overline{\mathrm{K}} \mathrm{N}$ channel $[6,7,10,12]$, whereas the phenomenological potentials models expect the existence of a single pole of the scattering amplitude at $1405 \mathrm{MeV}$ $[8,9,13]$.

The position of the $\Lambda(1405)$ is related to the strength of the $\bar{K} N$ potential which also determines the possible formation of more exotic $\mathrm{K}^{-}$multi-nucleon clusters. The lightest kaonic bound state is the di-baryonic $\mathrm{K}^{-}$pp cluster (and the isospin partner $\overline{\mathrm{K}}^{0} \mathrm{np}$ ), whose main decay channels are the $\Lambda \mathrm{p}$ and $\Sigma^{0} \mathrm{p}$ final states. The theoretical expectations about the position of this state are controversial, delivering a wide range of binding energies and widths [16], and the experimental findings are also contradictory $[17,18]$. To obtain a deeper understanding and to interpret the ambiguity between the available results, exclusive measurements from other experiments are still necessary. The authors of Refs. [19] stressed that the search of the kaonic nuclear clusters in $\mathrm{K}^{-}$induced reactions has to be performed with a clear knowledge of the yields and kinematic shapes of the $\mathrm{K}^{-}$multi-nucleon absorptions, since they overlap over a broad range of the phase space with the bound states.

In Section 2 the features of the DAФNE accelerator and the KLOE detector are summarized. In Section 3 the event selection procedure is described. Sections 4 and 5 are dedicated to the obtained results, and ongoing analyses, regarding $\mathrm{K}^{-}$multi-nucleon absorption processes, $\mathrm{ppK}^{-}$states, resonant and non-resonant $\mathrm{Y} \pi$ production in light nuclei.

\section{The KLOE detector at DAФNE}

DA $\Phi$ NE (Double Anular $\Phi$-factory for Nice Experiments) is a double ring $e^{+} e^{-}$collider, designed to work at the center of mass energy of the $\phi$ particle; the $\phi$ meson decay produces charged kaons with low momentum $(\simeq 127 \mathrm{MeV} / \mathrm{c}$ ) which is ideal to either stop them, or to explore the products of their low-energy nuclear absorptions.

The KLOE detector is centered around the interaction region of DAФNE, it consists of a large cylindrical Drift Chamber (DC) [22] and a fine sampling lead-scintillating fibers calorimeter [23], all immersed in the axially simmetric magnetic field with intensity of $0.52 \mathrm{~T}$, provided by a superconducting solenoid. The DC entrance wall composition is $750 \mu \mathrm{m}$ of carbon fibre and $150 \mu \mathrm{m}$ of aluminum foil. Dedicated GEANT Monte Carlo simulations of the KLOE apparatus show that out of the total 
number of kaons interacting in the DC entrance wall, about $81 \%$ are absorbed in the carbon fibre component and remaining $19 \%$ in the aluminum foil. The KLOE DC is filled with a mixture of helium and isobutane $\left(90 \%\right.$ in volume ${ }^{4} \mathrm{He}$ and $10 \%$ in volume $\mathrm{C}_{4} \mathrm{H}_{10}$ ). The chamber is characterized by excellent position and momentum resolutions. Tracks are reconstructed with a resolution in the transverse $R-\phi$ plane $\sigma_{R \phi} \sim 200 \mu \mathrm{m}$ and a resolution along the $z$-axis $\sigma_{z} \sim 2 \mathrm{~mm}$. The transverse momentum resolution for low momentum tracks $((50<p<300) \mathrm{MeV} / \mathrm{c})$ is $\frac{\sigma_{p_{T}}}{p_{T}} \sim 0.4 \%$. The calorimeter is composed of a cylindrical barrel and two endcaps, providing a solid angle coverage of $98 \%$. The volume ratio (lead/fibers/glue $=42: 48: 10)$ is optimized for a high light yield and a high efficiency for photons in the range (20-300) $\mathrm{MeV} / \mathrm{c}$. The photon detection efficiency is $99 \%$ for energies larger than $80 \mathrm{MeV}$ and it falls to $80 \%$ at $20 \mathrm{MeV}$ due to the cutoff introduced by the ADC and TDC thresholds. The position of the clusters along the fibers can be obtained with a resolution $\sigma_{\|} \sim 1.4 \mathrm{~cm} / \sqrt{E(\mathrm{GeV})}$. The resolution in the orthogonal direction is $\sigma_{\perp} \sim 1.3 \mathrm{~cm}$. The energy and time resolutions for photon clusters are given by $\frac{\sigma_{E}}{E_{\gamma}}=\frac{0.057}{\sqrt{E_{\gamma}(\mathrm{GeV})}}$ and $\sigma_{t}=\frac{57 \mathrm{ps}}{\sqrt{E_{\gamma}(\mathrm{GeV})}} \oplus 100 \mathrm{ps}$.

The AMADEUS experiment is conceived to integrate the high acceptance and momentum resolution KLOE detector with the low momentum $\mathrm{K}^{-}$beam of the DAФNE collider in a future dedicated setup. As a step zero, the data collected by the KLOE collaboration during the 2004-2005 data taking, corresponding to about $1.74 \mathrm{fb}^{-1}$ of total integrated luminosity, are analysed. The KLOE detector is used as an active target, the hadronic interaction of negative kaons with the materials of the apparatus being investigated.

\section{Particle Identification}

In the following the investigation of the $\mathrm{K}^{-}$interactions with the KLOE materials, followed by the $\Sigma^{0} \mathrm{p}, \Lambda \mathrm{t}$ and $\Lambda \pi^{-}$correlated productions in the final state, will be presented. The first step of the events selection is the $\Lambda(1116)$ identification, which proceeds through the reconstruction of the $\Lambda \rightarrow p+\pi^{-}$ $(\mathrm{BR}=63.9 \pm 0.5 \%)$ decay vertex. A resolution better than $1 \mathrm{~mm}$ for the $\Lambda$ decay vertex inside the DC volume is found based on Monte Carlo simulations. The mean value for the $\mathrm{p} \pi^{-}$invariant mass $\left(\mathrm{M}_{\mathrm{p} \pi^{-}}\right)$ is $(1115.753 \pm 0.002) \mathrm{MeV} / \mathrm{c}^{2}$ and the resolution is $\sigma=0.5 \mathrm{MeV} / \mathrm{c}^{2}$. Only statistical error is given, the systematics is under evaluation. The distribution of the $\Lambda$ decay vertex position in the radial coordinate $\left(\rho_{\Lambda}\right)$, shown in Fig. 1, exhibits the topology of the $\mathrm{K}^{-}$absorptions in KLOE. Four components are distinguishable, from inside to outside the $\mathrm{K}^{-}$absorptions occur: in the DAФNE beryllium sphere ( $5 \mathrm{~cm})$, the DA $\Phi$ NE aluminated beryllium pipe $(\sim 10 \mathrm{~cm})$, the KLOE DC entrance wall $(\sim 25 \mathrm{~cm})$ and the long tail originated from $\mathrm{K}^{-}$interactions in the gas filling the $\operatorname{KLOE~DC~}(25-200 \mathrm{~cm})$.

The identification of charged particles is performed using both the $d E / d x$ information from the DC wires and the measurement of the energy released in the electromagnetic calorimeter (EMC), as described in [1]. $\Sigma^{0}$ particles are reconstructed through the electromagnetic decay into $\Lambda \gamma$. The photon is identified using the energy released in the KLOE EMC [20, 21]. The position of the $\mathrm{K}^{-}$ absorption vertex (hadronic vertex) is defined as the vertex between the hyperon path and a correlated particle (proton, triton and negatively charged pion) track. As an example, the obtained resolution on the radial coordinate $\left(\rho_{\Lambda \mathrm{p}}\right)$ for the $\Lambda \mathrm{p}$ vertex is $1.2 \mathrm{~mm}$. Cuts on the absorption vertex radial position were optimised, based on MC simulations and a study of the $\Lambda$ decay path, to select $\mathrm{K}^{-}$absorptions in the various targets with minimal contamination from other components. More details on the particle identification procedure can be found in [18].

\section{$4 \mathrm{~K}^{-}$multi-nucleon absorptions measurements}

The $\Sigma^{0} \mathrm{p}$ correlated production was analysed in Ref. [18], selecting the $\mathrm{K}^{-}$captures in ${ }^{12} \mathrm{C}$ nuclei. The goal of the analysis was to pin down the contribution of the $\mathrm{K}^{-}$two-nucleon absorptions (2NA), in 


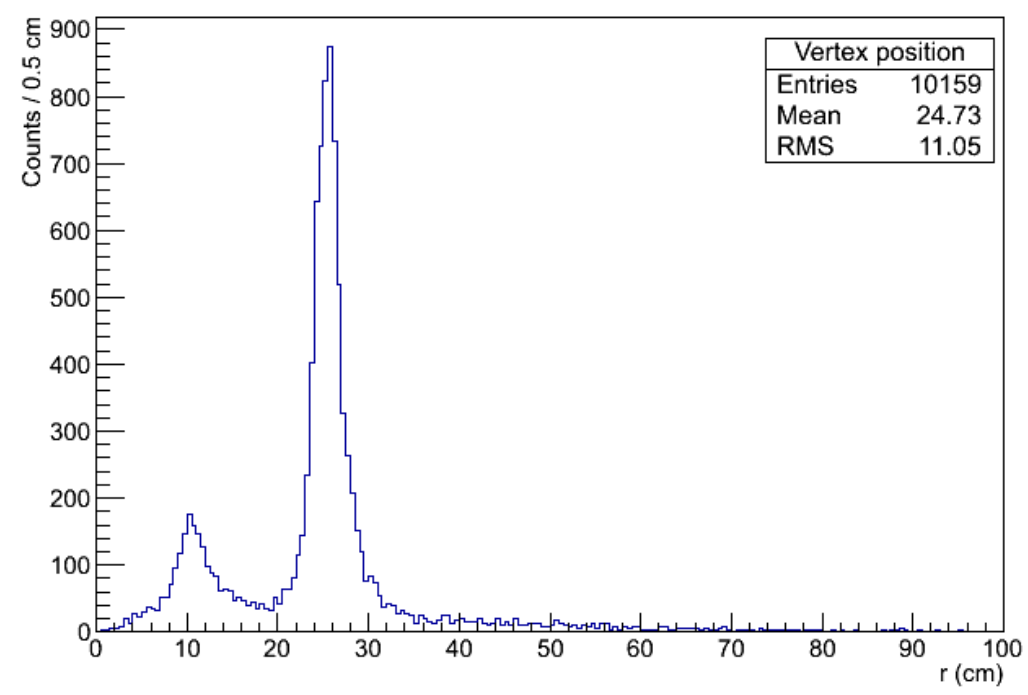

Figure 1. Radial position distribution $\rho_{\Lambda}$, of the $\Lambda$ decay vertex, for 2004-2005 KLOE collected data.

order to measure the yield per stopped $\mathrm{K}^{-}$, and to search for a possible contribution due to the $\mathrm{K}^{-} \mathrm{pp}$ bound state formation, decaying into the $\Sigma^{0} \mathrm{p}$ channel.

A simultaneus fit of the $\Sigma^{0} \mathrm{p}$ invariant mass $\left(\mathrm{M}_{\Sigma^{0}} p\right)$, the angular correlation $\left(\cos \left(\theta_{\Sigma^{0} \mathrm{p}}\right)\right)$, the $\Sigma^{0}$ and the proton momenta $\left(\mathrm{P}_{\Sigma^{0}}\right.$ and $\left.\mathrm{P}_{p}\right)$ was performed using the following contributing processes:

- $\mathrm{K}^{-} \mathrm{A} \rightarrow \Sigma^{0} \mathrm{p}_{\text {spec }} \pi \mathrm{A}^{\prime}(1 \mathrm{NA})$,

- $\mathrm{K}^{-} \mathrm{pp} \rightarrow \Sigma^{0} \mathrm{p}(2 \mathrm{NA})$,

- $\mathrm{K}^{-} \mathrm{ppn} \rightarrow \Sigma^{0} \mathrm{pn}(3 \mathrm{NA})$,

- $\mathrm{K}^{-} \mathrm{ppnn} \rightarrow \Sigma^{0}$ pnn (4NA).

The final state interactions (FSI) of the $\Sigma^{0}$ and $\mathrm{p}$ with the residual nucleus emerging from a $\mathrm{K}^{-} \mathrm{pp}$ capture in Carbon were taken into account. The fit is shown in Fig 2.

The yield of the 2NA, when the produced $\Sigma^{0}$ and p particles are free form any FSI process (2NA$\mathrm{QF})$, was measured for the first time, with good precision. More difficult is to disentangle the 3NA from 2NA + FSI processes due to their similar expected shapes. The obtained results are summarized in Table 4. A second fit was performed including a $\mathrm{K}^{-}$pp component, decaying into $\Sigma^{0} \mathrm{p}$. A systematic scan of possible binding energies and widths, varying within $(15 \div 75) \mathrm{MeV}$ and $(30 \div 70) \mathrm{MeV} / \mathrm{c}^{2}$ respectively, was performed. The best fit resulted in a binding energy of $45 \mathrm{MeV}$ and a width of $30 \mathrm{MeV} / \mathrm{c}^{2}$. The resulting yield normalised to the number of stopped $\mathrm{K}^{-}$is $\mathrm{K}^{-} \mathrm{pp} / \mathrm{K}_{\text {stop }}^{-}=(0.044 \pm$ 0.009 stat $\left._{-0.005}^{+0.004} \mathrm{syst}\right) \times 10^{-2}$. The significance of the bound state with respect to a statistical fluctuation was checked by means of an F-test. The significance of the $\mathrm{K}^{-} \mathrm{pp}$ contribution was found to be $1 \sigma$, which is not enough to claim the measurement of a bound state signal [18].

The study of the $\Lambda$ t correlated production following the $\mathrm{K}^{-}$capture in the DC gas is presently ongoing. The goal of this analysis is to measure the, extremely rare, 4NA absorption process of the $\mathrm{K}^{-}$, selecting the captures of the negatively charged kaons on Helium $\left(\mathrm{K}^{-}+{ }^{4} \mathrm{He} \rightarrow \Lambda \mathrm{t}\right)$. Some $\Lambda$ t events were identified in $[24,25]$ but the 4 NA contribution was not extracted. In our work the 

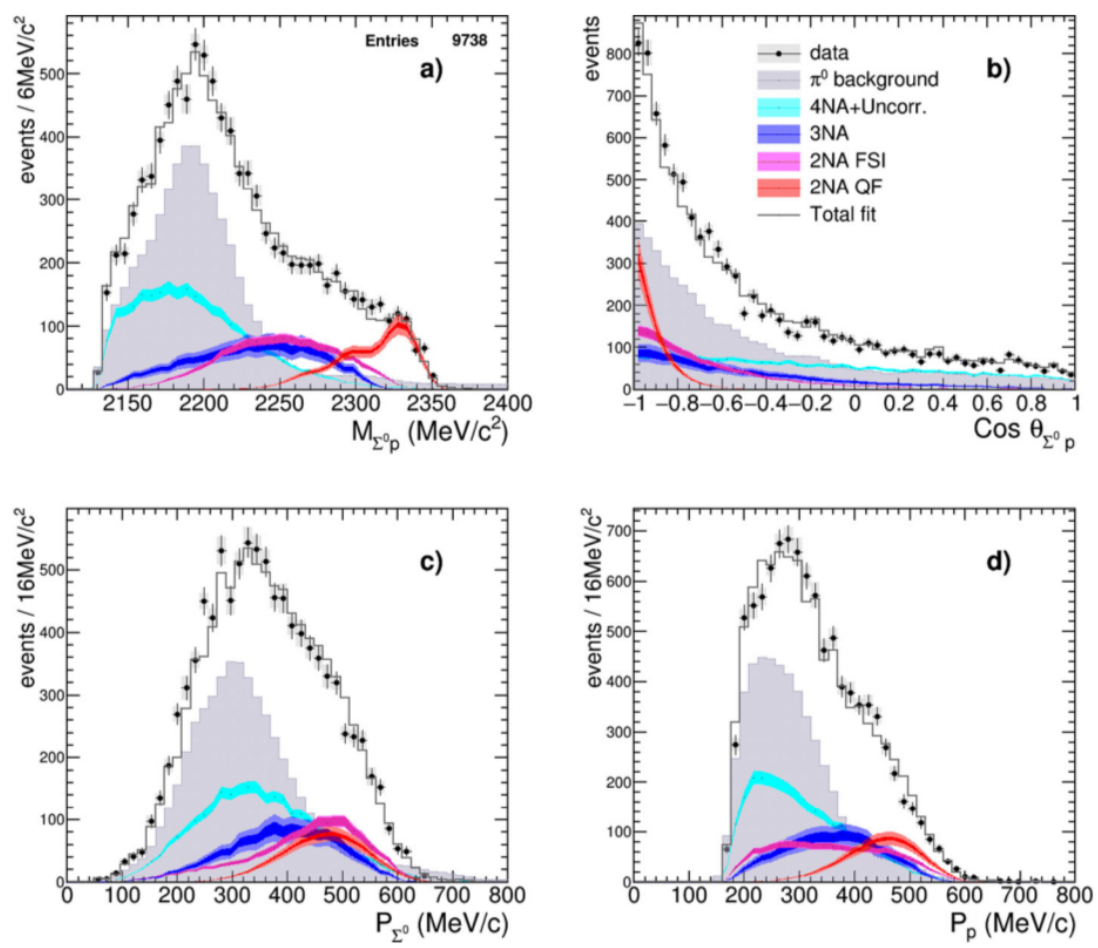

Figure 2. Fit of the $\Sigma^{0} p$ invariant mass, $\cos \left(\theta_{\Sigma^{0}}\right), \Sigma^{0}$ and proton momenta is shown. Data points are represented by black circles, the systematic errors by boxes, coloured distributions correspond to the simulated processes (light-coloured bands show the statistical errors and the darker bands represent the symmetrised systematic errors). The gray line is the total fit.

\begin{tabular}{cccc}
\hline Process & yield $/ \mathrm{K}_{\text {stop }}^{-} \times 10^{-2}$ & $\sigma_{\text {stat }} \times 10^{-2}$ & $\sigma_{\text {syst }} \times 10^{-2}$ \\
\hline 2NA-QF & 0.127 & \pm 0.019 & ${ }_{-0.000}^{+0.004}$ \\
2NA-FSI & 0.272 & \pm 0.028 & ${ }^{+0.022}$ \\
Tot 2NA & 0.399 & \pm 0.033 & -0.023 \\
3NA & 0.274 & \pm 0.023 \\
Tot 3 body & 0.546 & \pm 0.074 & -0.032 \\
4NA + bkg. & 0.773 & \pm 0.044 \\
\hline
\end{tabular}

Table 1. Production probability of the $\Sigma^{0} \mathrm{p}$ final state for different intermediate processes normalised to the number of stopped $\mathrm{K}^{-}$in the DC wall. The statistical and systematic errors are shown as well [18].

highest statistics ever of correlated $\Lambda$ t production was evidenced (150 events). The preliminary $\Lambda \mathrm{t}$ invariant mass and angular correlation distributions are shown in Fig. 3 left and right respectively. The signature of $\mathrm{K}^{-}$4NA in ${ }^{4} \mathrm{He}$ is the production of back-to-back $\Lambda \mathrm{t}$ pairs, with the highest energy permitted by the kinematics. Such events are represented in red in Fig. 3, and correspond to the cut $\cos \theta_{\Lambda \mathrm{t}}<-0.95$. This analysis is presently under finalization. 

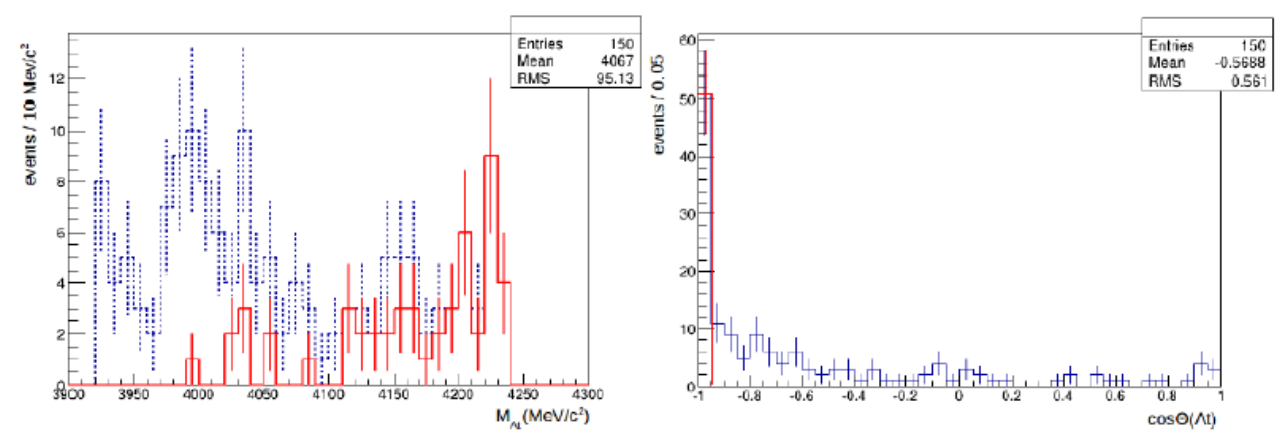

Figure 3. (Colour online.) $\Lambda t$ invariant mass (left) and $\cos \theta_{\Lambda t}$ (right). The events corresponding to the $\cos \theta_{\Lambda t}<$ -0.95 selection are shown in red.

\section{$5 \Lambda \pi^{-}$resonant and non resonant production}

The possibility to produce $\overline{\mathrm{K}}$ multi-N bound states is strongly related to the strength of the $\overline{\mathrm{K}} \mathrm{N}$ attractive interaction, which also determines the position of the $\Lambda(1405)$ state. In $\mathrm{K}^{-}$induced reactions, the $(\Sigma \pi)^{0}$ invariant mass resonant shape is influenced by the kinematic energy limit, imposed by the last nucleon binding energy. This limit is at about $1412 \mathrm{MeV} / \mathrm{c}^{2}$ and $1416 \mathrm{MeV} / 2$, for $\mathrm{K}^{-}$capture at-rest in ${ }^{4} \mathrm{He}$ and ${ }^{12} \mathrm{C}$ respectively. A second bias is represented by the non-resonant $\Sigma \pi$ formation, which gives rise to highly correlated hyperon-pion pairs, with narrow (of the order of $10 \mathrm{MeV}$ ) $\mathrm{M}_{\Sigma \pi}$ invariant masses spectra peaked just below the kinematic limit, as shown in Ref. [26]. The $\Lambda \pi$ and $\Sigma \pi$ non resonant production, for $\mathrm{K}^{-}$capture in light nuclear targets was never measured.

The study of the $\Lambda \pi^{-}$correlated production for $\mathrm{K}^{-}$captures in ${ }^{4} \mathrm{He}$ is presently ongoing. The goal is to measure the $\mathrm{I}=1$ contribution of the non-resonant $\mathrm{Y} \pi$ production $\left(\mathrm{K}^{-} \mathrm{n} \rightarrow \Lambda \pi^{-}\right)$, for the first time. The advantage to investigate the $\mathrm{I}=1$ channel is represented by the precise knowledge of the $\Sigma^{-}(1385)$ resonant transition amplitude. The $\Lambda$ and $\pi^{-}$kinematic distributions for $\mathrm{K}^{-}$captures in ${ }^{4} \mathrm{He}$, both at-rest and in-flight, were calculated in Ref. [27]. The momentum probability distribution functions, of the emerging hyperon pion-pairs, following $\mathrm{K}^{-} \mathrm{n}$ absorptions, are expressed in terms of the $\mathrm{K}^{-} \mathrm{n}$ transition amplitudes: the isospin $I=1 \mathrm{~S}$-wave non- resonant amplitude $\left(\left|f^{\mathrm{nr}}\right|\right)$ and the resonant $I=1$ P-wave amplitude, dominated by the $\Sigma^{-}(1385)$. Since the resonant amplitude is well known, the measured total momentum distributions can be used to extract the non-resonant $\left|f^{\text {nr }}\right|$ amplitude module below the $\overline{\mathrm{K} N}$ threshold.

Such measurement will also help to characterize the corresponding $(\Sigma \pi)^{0} I=0$ non-resonant transition amplitude, with the aim to extract the $\Lambda(1405)$ resonant shape.

\section{Acknowledgement}

We acknowledge the KLOE Collaboration for their support and for having provided us the data and the tools to perform the analysis presented in this paper. We acknowledge the CENTRO FERMI - Museo Storico della Fisica e Centro Studi e Ricerche "Enrico Fermi", for the project PAMQ. Part of this work was supported by the Austrian Science Fund (FWF): [P24756-N20]; Austrian Federal Ministry of Science and Research BMBWK 650962/0001 VI/2/2009; the Grantin-Aid for Specially Promoted Research (20002003), MEXT, Japan; the Croatian Science Foundation, under project 1680; Minstero degli Affari Esteri e della Cooperazione Internazionale, Direzione Generale per la Promozione del Sistema Paese (MAECI), Strange Matter project; Polish National Science Center through grant No. UMO-2016/21/D/ST2/01155. 


\section{References}

[1] C. Curceanu, K. Piscicchia et al., Acta Phys. Polon. B46 1, 203-215, (2015).

[2] R. Baldini et al., Proposal for a Phi-Factory, report LNF-90/031(R) (1990).

[3] F. Bossi, E. De Lucia, J. Lee-Franzini, S. Miscetti, M. Palutan and KLOE coll., Riv. Nuovo Cim. 31 (2008) 531-623

[4] N. Kaiser, P. Siegel, and W. Weise, Nucl. Phys. A594, 325 (1995).

[5] E. Oset and A. Ramos, Nucl. Phys. A635, 99 (1998).

[6] J. Oller and U. G. Meißner, Phys. Lett. B500, 263 (2001).

[7] T. Hyodo and D. Jido, Prog. Part. Nucl. Phys. 67, 55 (2012).

[8] Y. Akaishi, T. Yamazaki, Phys. Rev. C65, 044005 (2002).

[9] N. V. Shevchenko, Phys. Rev. C85, 034001 (2012).

[10] T. Hyodo and W. Weise, Phys. Rev. C77, 035204 (2008).

[11] S. Ohnishi, Y. Ikeda, T. Hyodo, and W. Weise, Phys. Rev. C 93, 025207 (2016).

[12] D. Jido, J. Oller, E. Oset, A. Ramos, and U. Meißner, Nucl. Phys. A725, 181 (2003).

[13] J. Esmaili et al., Phys. Lett. B 686 (2010) 23-28

[14] C. Patrignani et al. (Particle Data Group), Chin. Phys. C, 40, 100001 (2016).

[15] T. Hyodo, D. Jido, Prog. Part. Nucl. Phys. 67 (2012) 55.

[16] T. Yamazaki, et al., Phys. Rev. C 76045201 (2007)

A. Doté, et al., Phys. Rev. C 79014003 (2009)

S. Wycech, et al., Phys. Rev. C 79014001 (2009)

N. Barnea, et al., Phys. Lett. B 712132 (2012)

N.V. Shevchenko, et al., Phys. Rev. Lett. 98082301 (2007)

Y. Ikeda, et al., Phys. Rev. C 79035201 (2009)

E. Oset, et al., Nucl. Phys. A 881127 (2012)

P. Bicudo, Phys. Rev. D 76 031502(R) (2007)

J. Revai et al., Phys. Rev. C 90034004 (2014)

S. Maeda et al., Proc. Jpn. Acad. B 89418 (2013)

M. Bayar and E. Oset, Nucl. Phys. A 914349 (2013).

[17] M. Agnello et al. [FINUDA collaboration], Phys. Rev. Lett. 94, 212303 (2005)

G. Bendiscioli et al., Nucl. Phys. A 789 (2007) 222-242

T. Yamazaki et al. [DISTO collaboration], Phys. Rev. Lett. 104, 132502 (2010)

Y. Ichikawa et al. [E27 collaboration], Prog. Theor. Exp. Phys. 2015021 D01 (2015)

Y. Sada, et al., PTEP 2016 (2016) no.5, $051 D 01$

A. O. Tokiyasu et al. [LEPS collaboration], Phys. Lett. B 728, 616 (2014)

G. Agakishiev et al. [HADES collaboration], Phys. Lett. B 742, 242 (2015).

[18] O. Vazquez Doce, et al., Phys. Lett. B 758, (2016) 134.

[19] V. K. Magas, E. Oset, A. Ramos and H. Toki, Phys. Rev. C 74, 025206 (2006)

V.K. Magas, E. Oset, A. Ramos, Phys.Rev. C77 (2008) 065210

[20] K. Piscicchia et al., PoS Bormio2013 034 (2013)

[21] A. Scordo et al., PoS Bormio2014 039 (2014)

[22] M. Adinolfi et al., [KLOE Collaboration], Nucl. Inst. Meth. A 488, (2002) 51.

[23] M. Adinolfi et al. [KLOE Collaboration], Nucl. Inst. Meth. A 482, (2002) 368.

[24] R. Roosen, J. H. Wickens, Il Nuovo Cim. A Series 11, 66101 (1981)

[25] FINUDA collaboration, Phys. Lett. B, 669229 (2008)

[26] R. Del Grande et al., Acta Phys.Polon. B48 (2017) 1881 
[27] K. Piscicchia, S. Wycech, C. Curceanu, Nucl. Phys. A 954, 75-93 (2016) 